\title{
Nonadiabatic Dynamics in Semiquantal Physics
}

\author{
Mason A. Porter \\ Center for Applied Mathematics \\ Cornell University \\ April, 2001 \\ Revised July, 2001
}

\begin{abstract}
Every physical regime is some sort of approximation of reality. One lesserknown realm that is the semiquantal regime, which may be used to describe systems with both classical and quantum subcomponents. In the present review, we discuss nonadiabatic dynamics in the semiquantal regime. Our primary concern is electronic-nuclear coupling in polyatomic molecules, but we discuss several other situations as well. We begin our presentation by formulating the semiquantal approximation in quantum systems with degrees-
\end{abstract}


of-freedom that evolve at different speeds. We discuss nonadiabatic phenomena, focusing on their relation to the Born-Oppenheimer approximation. We present several examples-including Jahn-Teller distortion in molecules and crystals and the dynamics of solvated electrons, buckyballs, nanotubes, atoms in a resonant cavity, SQUIDs, quantum particle-spin systems, and micromasers. We also highlight vibrating quantum billiards as a useful abstraction of semiquantal dynamics.

\section{PAC NOS 03.65.Sq, 02.70.Ns, 05.45.-a, 05.45.Mt 1 Introduction}

Every physical regime approximates reality in some form or another. In continuum mechanics, one ignores the fact that a solid or fluid is composed of a finite number of discretely spaced particles because it is not necessary to consider this at the scale under consideration. In classical mechanics, one does consider discrete objects, but quantities such as energy and light are permitted to vary continuously. Indeed, there are so many photons in this regime that one would not notice the ensuing difference in illumination were a single one removed. In quantum physics, these quantities are treated as discrete-they have been quantized-and one expresses concepts such as position and momentum as operators rather than simply vectors. This regime is an approximation of the even more finely grained domain of quantum field theory, which may be in turn an approximation of even more intricate theories. Despite this outline, the picture is far from complete, as there are several regimes not mentioned above as well as others that lie at the 
borders between the regimes discussed above. For example, between the fully quantum regime and the classical one lie the semiclassical, quasiclassical, and semiquantal regimes, which-despite their nomenclature-are not the same. The semiclassical regime is a well-studied physical approximation to quantum mechanics. It is the domain of methods such as the WKB approximation and quantum chaology, the study of the quantum signatures of classical chaos.t One obtaines a semiclassical description from the fully quantal theory by taking the well-defined asymptotic limit $\hbar \longrightarrow 0$.

Less studied than the semiclassical regime is the semiquantal one. This latter description of physics has been analyzed far less than the semiclassical one in part because nobody has found a completely satisfactory asymptotic procedure to pass from the fully quantized regime to the semiquantal one. Nevertheless, there are several situations for which semiquantal physics is appropriate. Such systems are characterized by a mixture of classical and quantum physics. One may obtain a semiquantal description, for example, by coupling a classical system to a quantum-mechanical one. Moreover, semiquantal systems arise naturally when one uses the adiabatic or Born-Oppenheimer approximation, 3 which provides a widely accepted procedure for dividing a quantum-mechanical system into slow and fast subsystems. One begins this approximation by quantizing the fast subsystem, which consists of the electronic degrees-of-freedom (in the language of chemical physics). If one obtains well-separated energy levels, then one may also quantize the slow subsystem, which consists of the nuclear degrees-of-freedom (which can be either vibrational or rotational). If, however, the electronic eigenenergies of a $d$-state system are close to each other, then one ignores the rest of the 
spectrum, thereby obtaining a system described by $d$ electronic energy levels (each of which corresponds to the full contribution of a single eigenstate) that are coupled to a multitude of nuclear states. The semiquantal approximation consists of modeling these nuclear states as a continuum. That is, we treat the nuclear degrees-of-freedom of the present system as classical degrees-of-freedom, thereby obtaining a system with coupled classical and quantum components.

This breakdown of the Born-Oppenheimer approximation is a hallmark of nonadiabatic phenomena, which are important in the study of inelastic atomic and molecular collisions as well as in bound states of molecular systems. In particular, the Born-Oppenheimer approximation breaks down in exactly this manner for excited electron states of polyatomic molecules-often as a result of their symmetries. The near-degeneracy (and sometimes exact degeneracy) of several states is a common phenomenon in molecules-especially at higher energies. Both the energy spectrum and intramolecular dynamics can vary substantially from those observed during adiabatic behavior. it is not easy to incorporate nonadiabatic behavior into simple models of molecular dynamics, in which the canonical portrait of nuclear motion is described on a single well-defined surface of potential energy near the electronic degeneracy. Consequently, it is important to develop a semiquantal description of such systems that incorporates essential features of the nonadiabatic coupling.

The purpose of the present paper is to give an elementary presentation of the semiquantal approximation and some systems for which it is relevant. We focus on problems in which chaotic behavior can occur. We include 
few calculations and instead provide references to papers and monographs that include them. We begin our discussion by framing semiquantal physics in the context of the Born-Oppenheimer approximation before going into a more detailed discussion of nonadiabaticity. We then discuss polyatomic

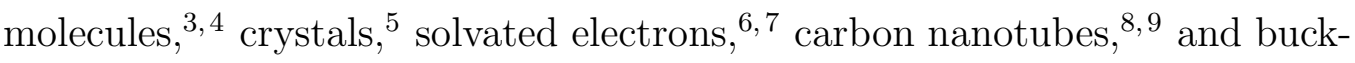
yballs.1010 These systems may be abstracted mathematically in terms of vibrating quantum billiards, 12,13 which are amenable to a semiquantal description because the boundaries are classical and the enclosed particles are quantum-mechanical 14 We also survey other systems such as a two-level system interacting with the electromagnetic field of a laser cavity, 15 micromasers, 16 quantum particle-spin systems, 17 SQUIDs, 18 and nuclear collective motion. 19

\section{The Semiquantal Approximation}

At issue in nonadiabatic analysis is the exent to which "classical path" (that is, functional integration 20) descriptions are relevant. In other words, one must consider how reasonable is it to treat the nuclear (slow, heavy particle) degrees-of-freedom classically in an effective potential determined by the quantum dynamics of the electronic (fast, light particle) degrees-offreedom. If one uses a path integral formulation of nonadiabatic scattering amplitudes, one obtains a formal solution to this problem.21,22 The exact (semiclassical) effective "potential" is nonlocal in time, so it must be computed iteratively. Consequently, practical computations of this quantity are almost impossible without approximations. Two ways of dealing with this 
are the so-called "surface-hopping" approach and the idea of self-consistent

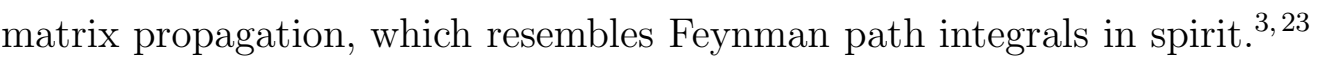

Consider a system with nuclear degrees-of-freedom $Q$ and electron degreesof-freedom $q$. The quantum dynamics of the full system may be expressed in integral form with a propagatorl (i.e., Green's function) $K$ as the kernel of the following integral equation:

$$
\psi\left(q^{\prime}, Q^{\prime}, t^{\prime}\right)=\int d q d Q K\left(q^{\prime}, Q^{\prime}, t^{\prime} \mid q, Q, t\right) \psi(q, Q, t) .
$$

One may equivalently expand $\psi$ in a basis of electronic states $\left\{\varphi_{n}(q)\right\}$ to obtain the equation

$$
\psi(q, Q, t)=\sum_{n} \chi_{n}(Q, t) \varphi_{n}(q, t),
$$

where the dynamics of the nuclear wavefunctions $\chi_{n}$ are determined using the reduced propagator $K_{\beta \alpha}$ :

$$
\chi_{\beta}\left(Q^{\prime}, t^{\prime}\right)=\sum_{\alpha} \int d Q K_{\beta \alpha}\left(Q^{\prime}, t^{\prime} \mid Q, t\right) \chi_{\alpha}(Q, t) .
$$

(Note that equation (2) is valid asymptotically (that is, adiabatically) only when the electronic and nuclear degrees-of-freedom can be separated from

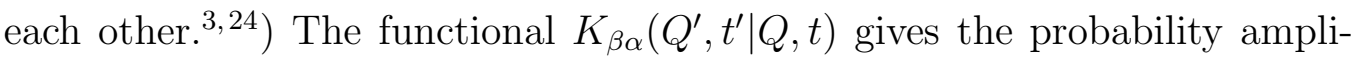
tude for the quantum system to go from state $\alpha$ to state $\beta$ as the nuclear variables move from $Q(t)$ to $Q^{\prime}\left(t^{\prime}\right)$. The probability of being in the electronic basis state $\beta$ at time $t^{\prime}$ is thus given by

$$
P_{\beta}\left(t^{\prime}\right)=\int d Q^{\prime}\left|\chi_{\beta}\left(Q^{\prime}, t^{\prime}\right)\right|^{2},
$$

where $\sum_{\beta} P_{\beta}\left(t^{\prime}\right)=1$ by conservation of probability. One may write the Green's function $K_{\beta}$ as a Feynman path integral, thereby expressing it as an 
integral over all paths $\bar{Q}(\bar{t})$ connecting the two endpoints $Q(t)$ and $Q^{\prime}\left(t^{\prime}\right)$. By assuming that the paths of the nuclear coordinates with the biggest contribution are those of stationary phase $\tilde{R}(t)$, one obtains a semiquantal approximation of the reduced propagator. One integrates only over "classical" (stationary phase) paths rather than over every path and obtains equations of motion that must be solved iteratively (due to temporal nonlocality). Indeed, the force on the trajectory at time $\bar{t}$ depends on both the forward and backward propagated wavefunctions, which can only be determined if one knows the full trajectory $\tilde{R}(t)$. One begins the iterative procedure by guessing a trajectory, which specifies the electronic component of the Hamiltonian. One then integrates the time-dependent Schrödinger equation both forwards and backwards from the appropriate boundary states and uses mixed-state solutions to determine the force on the trajectory.

If the electronic state $\alpha$ is initially occupied, the initial wave vector at the outset is given by the components

$$
\begin{gathered}
\chi_{i}(Q(t), t)=0 \quad \forall i \neq \alpha, \\
\chi_{\alpha}(Q(t), t)=f(Q(t)),
\end{gathered}
$$

where $f(Q(t))$ is the shape of the initial nuclear wavefunction on the electronic surface $\alpha$. Hence, the components of the wavefunction at time $t^{\prime}$ are given by

$$
\chi_{\beta}\left(Q^{\prime}, t^{\prime}\right)=\int d Q K_{\beta \alpha}\left(Q^{\prime}, t^{\prime} \mid Q, t\right) f(Q) .
$$

One subsequently uses a semiquantal approximation of the propagator $K_{\beta \alpha}$ in order to obtain the semiquantal expression for the advanced nuclear wavefunction at $Q^{\prime}$ moving over the electronic state $\beta$. In principle, one can obtain 
the stationary phase paths using a root search, as they are specified in terms of a boundary value problem. One considers initial velocities $d \tilde{Q} / d t$ and proceeds iteratively until one has found all convergent paths that reach the desired endpoint $Q^{\prime}$. Finally, one computes the wavefunction by integrating over all these path contributions from each initial point.

The surface-hopping method has been applied to scattering problems, for which nonadiabatic effects are usually localized. One assumes that a trajectory evolves on a single manifold of adiabatic potential surfaces for every nuclear configuration except those near electronic degeneracies. One then calculates the probability that the trajectory jumps to a nearby surface, on which the evolution proceeds adiabatically. Many systems, however, are constantly in regions of near-degeneracy, so one requires a dynamical description of nonadiabatic evolution. It is desirable, moreover, to have a scheme with which to analyze the nonadiabatic behavior of bound and quasibound molecular states. If the nuclei in such states are localized in regions of electronic degeneracy (or near-degeneracy), one may use effective-path methods that couple classical nuclear motion self-consistently with quantum electronic motion. 1 One may assume that the classical nuclear motion is determined by its interaction with the electronic system in a self-consistent manner. Using the vibrational and rotational (rovibrational) coupling terms in the molecular Hamiltonian, one obtains a time-dependent electronic Hamiltonian, which causes transitions in the molecule's electronic states because of its dependence on the nuclear degrees-of-freedom. Time-dependence in these electronic states leads to a time-dependent nuclear potential, because the molecular Hamiltonian depends on the nuclear coordinates. Simple examples of 
this sort of self-consistent coupling may be abstracted and studied as a vibrating quantum billiards, in which the enclosed particle (fast subsystem) is coupled to the surrounding wall (slow subsystem). 13 , 25 This abstraction is very useful, as it is easily generalized and may also be applied to the study of systems in chemical physics (such as polyatomic molecules, solvated electrons, Jahn-Teller distortions, and chemical nanostructures).

We now continue our discussion of path integral methods. Consider a system with one nuclear degree-of-freedom that includes a localized crossing of two adiabatic potentials. It is requisite that the two potentials have similar slopes in the crossing region and that the nuclear kinetic energy is large compared to the difference between the two potentials in the interaction region. 3 Moreover, one needs the electronic states to be close enough to each other energetically so that the nuclear degrees-of-freedom may be approximated as a continuum. The nuclear mass is assumed to be large relative to the electronic mass, so the nuclear energy levels are more finely grained than the electronic eigenenergies. If the nuclear energy levels are sufficiently close together, they are well-approximated by a continuum. The separation of these energy levels becomes smaller both as a result of larger nuclear masses and as a consequence of closeness of electronic energy levels. Hence, one requires some combination of sufficiently large nuclear mass and sufficiently degenerate electronic energy levels in order to approximate the nuclear degrees-of-freedom as classical.

Although this type of self-consistent coupling of classical and quantum dynamics has appeared often in the chemical physics literature, there remain conceptual difficulties and inconsistencies in the semiquantal approximation. 
Consider a system that is asymptotically in a two-state electronic superposition. The nuclei undergo some sort of averaged dynamical motion that does not correspond to that determined by either of the two adiabatic surfaces, despite the fact that one could argue by physical reasoning that the latter is the expected behavior. A way to surmount this difficulty is the classical electron picture, which facilitates treatment of resonant processes such as electronicvibrational and electronic-rotational energy transfer. ${ }^{26}$ This method has been applied successfully to several systems describing nonadiabatic collisions, including charge transfer in $N a+I$ collisions, the quenching of the fluorine atom $F^{*}\left({ }^{2} P_{\frac{1}{2}}\right)$ via collisions with $H^{+}$or $X e$, and collinear and three-dimensional systems. Fully quantal calculations are available for some of these systems, and the semiquantal analyses produce cross sections and transition probabilities that are consistent with these studies. Additionally, the semiquantal calculations provide a correct description of resonant features.

\section{The Born-Oppenheimer Approximation and Nonadiabatic Phenomena}

It is more difficult to find electron orbits in molecules than in atoms because the effective potential felt by the electrons is no longer well-approximated as spherically symmetric. One pictures the molecular nucleus as having classical equilibrium positions about which it slowly oscillates. The electrons travel rapidly around the nucleus and are affected by the oscillations of the latter. This perspective is effective because a nucleus (with mass $M$ ) is much more massive than electrons (each of which have mass $m$ ). The mass ratio 
$m / M$ is typically about

$$
\frac{m}{M} \approx 10^{-5} \text { or } 10^{-4},
$$

so the magnitude of the zero-point motion of the nucleus is far smaller than that of the electrons. (Zero-point motion describes the minimal motion due to Heisenberg's Uncertainty Principle.)

From the perspective of an electron, the nucleus is practically stationary. As long as the electronic energy levels are sufficiently far apart, the only effect of the slow nuclear vibrations is to adiabatically deform the electronic eigenstates. A molecule with typical radius $a$ has electrons with approximate momenta $\hbar / a$, so the energetic spacing of these electrons is about $\hbar^{2} / m a^{2}$.

From the nuclear point of view, the electrons are a blurry cloud. The electronic wavefunctions distort as the nuclei move, thereby causing small changes in the electronic energies. Additionally, the nuclei tend to move towards positions of minimum electronic energy, as if they were immersed in an elastic medium formed of electrons. 2 Molecular nuclei thus oscillate about energy minima, a phenomenon captured by the vibrating quantum billiard model of electronic-nuclear coupling. 3 One can estimate the frequency $\omega$ of nuclear oscillations by assuming that the nucleus resides in a harmonic potential $M \omega^{2} r^{2} / 2$, where $r$ is the displacement of the nucleus from equilibrium. If this displacement is given by the distance $a$, then the electronic energy experiences a change of about $\hbar^{2} / 2 m a^{2}$. As a rough approximation,

$$
\frac{M \omega^{2} a^{2}}{2} \approx \frac{\hbar^{2}}{2 m a^{2}},
$$

so the nuclear frequency is given by 4

$$
\omega \approx \sqrt{\frac{m}{M}} \frac{\hbar}{m a^{2}} .
$$


The nuclear vibration energies $\hbar \omega$ are consequently a factor of $\sqrt{m / M}$ smaller than the electronic excitation energies and are on the order of tenths or hundredths the size of an electron volt.

The zero-point nuclear energy in a harmonic potential is

$$
\frac{P^{2}}{2 M} \approx \frac{\hbar \omega}{2},
$$

so its corresponding zero-point momentum is

$$
P \approx\left(\frac{M}{m}\right)^{\frac{1}{4}} \frac{\hbar}{a},
$$

which is about ten times larger than the momentum of an electron. A typical nuclear velocity is thus

$$
v_{N}=\frac{P}{M} \approx\left(\frac{m}{M}\right)^{\frac{3}{4}} \frac{\hbar}{m a} .
$$

The nuclear deviation from equilibrium $\delta$ satisfies

$$
\frac{M \omega^{2} \delta^{2}}{2} \approx \frac{\hbar \omega}{2},
$$

so

$$
\left(\frac{\delta}{a}\right)^{2} \approx \frac{\hbar \omega}{M \omega^{2} a^{2}} \approx \frac{E_{N}}{E_{e}} \approx \sqrt{\frac{m}{M}},
$$

which implies that 2 年

$$
\left(\frac{\delta}{a}\right) \approx\left(\frac{m}{M}\right)^{\frac{1}{4}} \approx \frac{1}{10} .
$$

In addition to vibrations, one may consider the rotation of the entire molecule about its center of mass, although the energy due to such excitations is very small since the molecule does not experience much distortion as a 
result of this motion. If the angular momentum of the rotational motion is $\hbar l$, then its accompanying energy is

$$
E_{\text {rot }} \approx \frac{\hbar^{2} l(l+1)}{2 M a^{2}} \approx \frac{m}{M} E_{e}
$$

In general, a molecular excited state can be decomposed into electronic, vibrational, and rotational excitations. Two examples of vibrational motion are pulsing (as in vibrating quantum billiards 14 ) and "bouncing" of the centerof-mass (as has been proposed as a mechanism for energy transfer in buckyball 10 ). Together, the vibrational and rotational excitations comprise the nuclear (or rovibrational) contribution to the energy. The total energy is given by the sum of the contributions from its three components:

$$
E=E_{e}+E_{N}+E_{\text {rot }}
$$

Let us formalize the preceeding discussion (which is based on the presentation of Baym24). We supplement the above analysis by applying the Born-Oppenheimer approximation to the Schrödinger equation. This scheme provides a widely accepted procedure for dividing quantum systems into slow and fast subsystems. The first step in this approximation is to quantize the fast (electronic) subsystem. If this results in energy levels with sufficient separation (because the electronic energy levels are sufficiently far apart for the given nuclear and electronic masses), then one can also quantize the slow (nuclear) subsystem in order to perform a fully quantum analysis. This results in a familiar spectrum describing the coupling between the electronic and nuclear subsystems. (In the language of physical chemistry, we think of the fast system as describing particles such as electrons and the slow system as describing nuclear variables.) If, however, the electronic energy levels are 
sufficiently close together, the situation is more complicated. One uses a semiquantal approximation by modeling as a continuum the large number of vibrational states that are coupled to the $d$ electronic states. Such systems are $(d+s)$ degree-of-freedom $(d o f)$ Hamiltonian systems, where $d$ of the $d o f$ are quantum-mechanical and the other $s$ are classical.

The number of classical degrees-of-freedom of such molecular systems (in other words, the number of nuclear of) is known as the degree-of-vibration (dov) of the system. 13 In semiquantal systems, many of which may be abstracted mathematically as vibrating quantum billiards, one often observes a form of quantum chaos known as semiquantum chaos, where the nomenclature reflects the fact that it occurs in the semiquantal regime.14,25,27 Another form of quantum chaos, called quantized chaos or quantum chaology, is studied in the semiclassical and high quantum-number regimes.628 This latter behavior may be observed by fully quantizing the motion of the molecular systems we have been describing. Part of the value of the semiquantal setting is that one may observe chaos even in low energy systems, such as nuclei that have been coupled to two-level electronic systems consisting of the ground state and the first excited state of appropriate symmetry. In the setting of quantum chaology, one observes chaos only in states with high energy In other words, one must pass to the semiclassical or high quantum-number limits in order to observe chaotic behavior. The ground state is not encompassed by these limits, so the semiquantal regime is important for capturing chaotic dynamics of low-energy states. Such behavior has been observed experimentally B $^{3}$

Let us now consider the relation of the Born-Oppenheimer approximation 
to nonadiabatic phenomena. In so doing, we largely follow the presentation

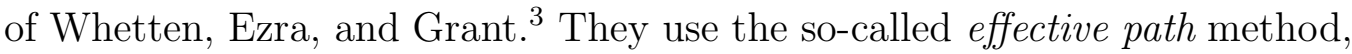
in which the electronic degrees-of-freedom are treated in the same manner as the nuclear degrees-of-freedom. One analyzes the nuclear motion classically to derive an effective Hamiltonian describing the evolution of the electronic states. An abstraction of this type of system is a vibrating quantum billiard, 3 in which the one derives evolution equations that may be treated as a classical Hamiltonian system. For a symmetric two-level system in that context, the dynamics of the quantum variables occur on the Bloch sphere.29 (Alternatively, one can describe the quantum dynamics of the system using action-angle variables.) After ignoring the quantum setting during numerical simulations, one must later interpret one's results in this context. This reinterpretation leads to interesting mathematical and physical phenomena. For example, bound states of molecules may exhibit nonadiabatic behavior. If the nuclei are localized near a degeneracy, effective path methods that couple classical nuclear motion with quantum-mechanical electronic motion provide a self-consistent (though possibly approximate) treatment of the effects of nonadiabatic coupling. One situation to which this has been applied is the Jahn-Teller $\mathrm{E} \times$ e system, in which a doubly degenerate electronic state is coupled to a doubly degenerate vibrational mode. 1

Important manifestations of nonadiabatic behavior may be observed in simple examples of symmetry-based electronic degeneracy. Without such degeneracy, one may approximate the molecular wavefunction using the BornOppenheimer (adiabatic) approximation. Using this scheme, the wavefunction is expressed as a product of electronic and nuclear wavefunction. One 
expands the wave in a $d$-dimensional electronic basis when one is near a $d$-fold degeneracy. Such degeneracies are common in the space spanned by nuclear (vibrational) coordinates.

The stationary and spinless Schrödinger equation for a single molecule is

$$
\left[T_{N}+H_{e}(q, Q)\right] \psi_{d}(q, Q)=E_{d} \psi_{d}(q, Q)
$$

where $T_{N}$ is the nuclear kinetic energy operator and

$$
H_{e}(q, Q) \equiv T_{e}+U_{e e}+U_{e N}+U_{N N}+V
$$

is the electronic Hamiltonian. Because of the coupling, $H_{e}$ depends (continuously) on the nuclear coordinates $Q$. Its components are the particle (electronic) kinetic energy $T_{e}$, the interelectron repulsion potential $U_{e e}$, the electron-nuclear attraction $U_{e N}$, the internuclear repulsion $U_{N N}$, and an external potential $V$. The nuclear kinetic energy $T_{N}$ is proportional to $1 / M$, so it is a small term in the total Hamiltonian. The Born-Oppenheimer scheme is to calculate the eigenenergies and eigenstates of the total molecular Hamiltonian by treating $T_{N}$ as a small perturbation whose expansion parameter is $(m / M)^{\frac{1}{4}}$, the ratio of nuclear vibrational displacement to the spacing between nuclei.

The molecular Hamiltonian $H$, given by

$$
H=T_{N}+H_{e}
$$

is the sum of its nuclear and electronic components. In the vibrating quantum billiard model, 13 an abstract example of a semiquantal system, the nuclear kinetic energy is simply the kinetic energy of the billiard boundary:

$$
T_{N} \equiv \frac{P^{2}}{2 M},
$$


where $P$ is the momentum of the boundary and $M$ is its mass. The only electronic Hamiltonians that have been considered in this abstract situation are ones without interelectron repulsion, electron-nuclear attraction, and internuclear repulsion. In other words, the particle's electronic Hamiltonian $H_{e}$ is given by the sum of its kinetic energy component $T_{e}$ and the external potential $V$ :

$$
H_{e} \equiv T_{e}+V
$$

where

$$
T_{e} \equiv K=-\frac{\hbar^{2}}{2 m} \nabla^{2}
$$

and $m$ is the mass of the confined particle. One would add an interelectron repulsion potential when considering a billiard with more than one enclosed particle. Similarly, one would add an electron-nuclear attraction term if considering vibrating quantum billiards with rebound.

The particle confined within the billiard is constrained to collide elastically against the billiard boundary, so one applies Dirichlet boundary conditions to the billiard walls of a priori unknown shape. (Such situations are known as free-boundary problems. 30) The billiard resides in a potential $V$, which adds a second component to the electronic Hamiltonian. Harmonic potentials have been considered most often, although quartic ones have been studied a bit as well 31 One observes bifurcations in the dynamics as one alters the potential. 11 In these studies, the potential $V$ depends on the vibrational coordinates $Q$, which represent the boundary components undergoing oscillations. (For the radially vibrating spherical quantum billiard, for example, the oscillating portion of the boundary is simply the radius a.) Moreover, 
when $V$ depends implictly on time (via the nuclear coordinates), it is considered part of the boundary conditions of the problem. In this situation, the Hamiltonian inserted into the Schrödinger equation is just the kinetic energy $K$. If, however, the potential $V$ depends explicitly on the spatial variables $(x, y, z)$ or on time, the Hamiltonian inserted into Schrödinger's equation is instead $K+V$. Finally, note that the electronic variables in vibrating quantum billiards may be parametrized by either action-angle variables or Bloch variables. $3,13,29$

In general, one may derive coupled vibrational equations in two different manners. One way is to use the so-called diabatic basis. The molecular wavefunction is given by

$$
\psi_{d}(q, Q)=\sum_{k} \varphi_{k}\left(q ; Q_{0}\right) \chi_{k}(Q)
$$

where the orthonormal electronic states $\varphi_{k}$ are calculated by solving the electronic Schrödinger equation at a chosen reference configuration $Q_{0}$ :

$$
H_{e}\left(Q_{0}\right) \varphi_{k}(q)=E_{k}^{0} \varphi_{k}(q)
$$

One then determines the vibrational wavefunctions $\chi_{k}$ by a set of coupled equations with Hamiltonian matrix elements given by

$$
H_{k k^{\prime}}=T_{N} \delta_{k k^{\prime}}+\left\langle\varphi_{k}\left|H_{e}(Q)\right| \varphi_{k^{\prime}}\right\rangle
$$

The nuclear kinetic energy $T_{N}$ is diagonal in this basis, yielding a condition that must be satisfied by any physical problem that the present analysis is purported to model. One continues to expand the matrix elements of the electronic Hamiltonian $H_{e}(Q)$ to obtain

$$
H_{k k^{\prime}}=\left(T_{N}+E_{k}^{0}+\Delta U_{N N}\right) \delta_{k k^{\prime}}+\left\langle\varphi_{k}\left|\Delta U_{e N}\right| \varphi_{k^{\prime}}\right\rangle
$$


so that each diagonal element defines an effective vibrational Hamiltonian. This Hamiltonian is the sum of the nuclear kinetic energy operator $T_{N}$ and the Hellman-Feynman potential for nuclear motion, 3 , 国 which contains a term from internuclear repulsions $U_{N N}$ as well as one from attraction to the electronic charge distribution $\varphi_{k} * \varphi_{k}$. Such a vibrational Hamiltonian neglects any response that the electronic state may have to the changing nuclear configuration. The off-diagonal coupling terms that have been neglected arise from the $\Delta U_{e N}$ term. Hence, it is the change in the potential describing electronic-nuclear attraction as a function of the changing nuclear configuration that induces mixing in diabatic basis states. This yields both adiabatic and nonadiabatic correlations of electronic and nuclear motion. 目

Alternatively, one may expand $\psi_{d}(q, Q)$ using a basis of adiabatic electronic states:

$$
\psi_{d}(q, Q)=\sum_{m} \varphi_{m}(q ; Q) \chi_{m}(Q)
$$

where $\varphi_{m}(q ; Q)$ is a solution of the electronic Schrödinger equation

$$
H_{e}(Q) \psi_{m}(q ; Q)=E_{m}(Q) \psi_{m}(q ; Q)
$$

which depends on the nuclear coordinates $Q$. This is the approach that has been followed in the study of vibrating quantum billiards. 1 , 国, 2 国 Equation (28) is the Born-Huang expansion, which consists of the Born-Oppenheimer expansion plus the diagonal nuclear nonadiabatic coupling. The electronic eigenvalues (which are different for different nuclear configurations) determine the adiabatic potential surfaces, which change with the nuclear configuration because of the dependence of the eigenenergies on the nuclear 
variables $Q$. One derives equations of motion for the adiabatic vibrational amplitudes $\chi_{m}$ in which the coupling is now due to the off-diagonal matrix elements of the nuclear kinetic energy.

Translating to mathematical language, we derive a $d$-mode Galërkin projection, 33 where the integer $d$ refers to the assumption of a $d$-level electronic system (corresponding to a $d$-fold near-degeneracy). For vibrating quantum billiards, this Born-Oppenheimer expansion corresponds to performing an eigenfunction expansion in the wavefunction as though one had a stationary boundary (such as a sphere of constant radius if one has spherical symmetry) and then reinserting the time-dependence in the resulting eigenstates and normalization factors. For example, this expansion would be performed using spherical Bessel functions if one were considering the radially vibrating sphere 13 We note that diabatic expansions are often more convenient for practical calculations because they correspond to fixed electronic states. Adiabatic expansions (and associated potential energy surfaces), on the other hand, arise naturally from quantum chemistry calculations and are also amenable to a dynamical systems approach.

The analysis of molecular bound states is a particular example relevant to the above discussion. In this situation, the adiabatic potential has minima corresponding to nuclear equilibria. 3 (This set of minima is not unique, because the system is invariant under spatial translations of the molecule as well as rotations about its center of mass.) To describe nuclear motion, one has to separate the vibrational degrees-of-freedoms from the translational and rotational ones. (This can be formalized mathematically as a reduction procedure.34) The potential energy has minima in the vibrational coordinates 
so obtained. One expands the potential about the equilibrium nuclear separations in order to calculate the energies associated with nuclear vibration. The vibrational part of the Schrödinger equation ultimately becomes a set of coupled harmonic oscillators, which one then studies using normal mode expansions.

Finally, note that geometric phase ("Berry phase") often occurs in the context of the Born-Oppenheimer approximation. For the present discussion, we (mostly) follow the presentation of Zwanziger, Koenig, and Pines.35 Consider a Hamiltonian with two or more variables that depend slowly on time. Thus, we require a $2+d o f$ Hamiltonian system in which at least one of the degrees-of-freedom is slow. Semiquantal problems such as those described in the present paper fit into this framework. At each instant, define a smoothly varying (and single-valued) basis of eigenstates $\left\{\left|\psi_{k}[x(t)]\right\rangle\right\}$ as solutions to the eigenvalue equation

$$
H[x(t)]\left|\psi_{k}[x(t)]\right\rangle=\lambda_{k}[x(t)]\left|\psi_{k}[x(t)]\right\rangle
$$

With the adiabatic approximation, a system beginning in the state $\left|\psi_{k}[x(0)]\right\rangle$ evolves to the state $\left|\psi_{k}[x(t)]\right\rangle \equiv\left|\psi_{k}[x(t)]\right\rangle$, which specifies the state at time $t$ up to a phase. However, one must still compute the phase at this time relative to that at time zero. In determining this phase, one must satisfy the relation

$$
\left\langle\psi_{n} \mid \frac{d \psi_{n}}{d t}\right\rangle=0
$$

which can be met at any specific time but which is not necessarily satisfied simultaneously at every point in space. 35 (In the language of geometry, equation (31) gives the requirement for parallel transport of the connection A, 
which is a gauge shift 34 37) That is, this choice in phase may be different at different points in space. Because of this complication in defining the phase of the basis $\{|\psi[x(t)]\rangle\}$ globally, there necessarily exists a phase factor due to the geometry of configuration space rather than simply the dynamical equations. In order to study such geometric phases, Sir Michael Berry considered a cyclic evolution of period $T$ in the position variables. The initial and final eigenspaces are hence the same, and the problem of comparing the phases at times 0 and $T$ is well-posed.

The eigenstate at time $T$ is given by

$$
\left|\psi_{n}[x(T)]\right\rangle=e^{-i \int_{0}^{T} \lambda_{n}[x(t)] d t} e^{i \gamma_{n}(C)}\left|\psi_{n}[x(0)]\right\rangle,
$$

where $C$ denotes the closed path traversed in configuration space. The dynamical phase is

$$
\varsigma_{n}=\int_{0}^{T} \lambda_{n}[x(t)] d t,
$$

and the geometric phase is $\gamma_{n}(C)$. Using the Schrödinger equation, we find that Berry's phase is given by

$$
\gamma_{n}(C)=\int_{C} A \cdot d x,
$$

where

$$
A=\left\langle\psi_{n} \mid i \nabla_{x} \psi_{n}\right\rangle
$$

is the gauge shift.

The geometric phase $\gamma_{n}(C)$, which is a real quantity, depends only on the initial eigenstate, the geometry of the path $C$, and whether or not $C$ 
surrounds a singularity. If the configuration space is simply connected and $\left|\psi_{n}[x(t)]\right\rangle$ may be smoothly chosen to be real-valued everywhere, then the geometric phase is zero. We remark that equation (34) involves the matrix element (35) that causes the adiabatic theorem (31) to break down. 35 One sees this immediately by applying the Chain Rule:

$$
\left\langle\psi_{m} \mid \frac{d \psi_{m}}{d t}\right\rangle=\left\langle\psi_{m} \mid \nabla_{x} \psi_{m} \cdot \frac{d x}{d t}\right\rangle=\left\langle\psi_{m} \mid \nabla_{x} \psi_{m}\right\rangle \cdot \frac{d x}{d t}
$$

Also note that one may derive Berry's phase using a path integral formulation. To consider the relevance of geometric phase to adiabatically evolving systems, we remove the $\exp \left[i \gamma_{n}(C)\right]$ factor via a gauge transformation. This adjusts our Hamiltonian by adding a vector potential term given by equation (35). A system with nonvanishing Berry phase may thus be treated using an effective Hamiltonian obtained via the transformation

$$
\nabla \mapsto \nabla-i A
$$

With equation (37), the geometric phase is absorbed into the dynamical phase $\varsigma_{n}$, even though its ultimate source is the geometry of configuration space.

The gauge potential just discussed is Abelian, like the one that occurs in electromagnetism.20 Although it occurs in an abstract space, one may still observe its effects. As in more familiar situations, it arises from ambiguities in the description of a system. In the present case, these ambiguities reflect the variety of manners in which one may select eigenstate phases. Such an ambiguity in assigning relative coordinates occurs whenever a problem may be separated into two subcomponents. In the present context, one has a natural separation into fast (electronic) and slow (nuclear) dynamics. 
In principle, one may therefore derive a geometric phase for any vibrating quantum billiard. Such analyses have already been performed for similar molecular models such as the $E \times e$ Jahn-Teller system. One computes a geometric phase $\gamma(C)$ of value $-\pi$, yielding a phase factor of -1 . Hence, if one specifies a basis of single-valued eigenstates, Berry's phase imposes a sign change and double-valued behavior. One may treat this example as a special case of a three-dimensional problem such as a spin $1 / 2$ particle in a planar magnetic field. (That is, the particle may move in three-space, but the field is planar.) The mathematical distinction between this perspective and the previous one is that analyzing the problem in two-dimensions forces a topological interpretation of the sign change-whereas considering a third dimension permits a geometric interpretation of the sign change. Interpreted topologically, the sign change arises from the fact that the configuration space $\left(\mathbb{R}^{2}-\{0\}\right)$ is not simply connected. Interpreted geometrically, the configuration space becomes curved because it deviates from the plane. In exchange, it is now simply connected. This geometric perspective illustrates a similarity between molecular spectroscopy and spin in a magnetic field. 35 Both the Jahn-Teller $E \times e$ problem and the spin $1 / 2$ particle in a planar magnetic field are described by real, symmetric $2 \times 2$ matrices, so the fundamental similarities between these systems are ultimately due to the commonlity of their Lie structure圂 One may think of the magnetic field as representing the semiclassical limit of a localized packet of nuclear configurations, and the corresponding molecular problem may be generalized to three dimensions by analyzing molecules whose nuclear configurations have higher symmetries. One may thus construct an analogy between vibrations in molecules and 
crystals and the problem of a spin 1/2 particle in a magnetic field. Additionally, one may construct analogies with other spin systems. For example, the triply degenerate electronic state of a $T \times(t+e)$ system is related to a spin 1 particle in combined magnetic and electric quadrupole fields. This idea of analyzing systems based on their common symmetries is a hallmark of the field of geometric mechanics, 34 which may prove very useful to the study of molecular dynamics as well.

\section{Molecules and Crystals}

In the present section, we discuss why polyatomic molecules are appropriately described semiquantally. We hinted at this description earlier, but we now consider it at length. Before beginning this discussion, however, it is important to recall that-in addition to the modern results reviewed in the present paper-molecules and crystals can also be studied using well-known techniques such as the Bloch theorem.8.83, 39

Earlier in this work, we noted that polyatomic molecules may be abstracted as vibrating quantum billiards. This abstraction arose initially by considering one-dimensional vibrating quantum billiards as a model for diatomic molecules.12 More generally, a $d$-mode Galërkin expansion of a vibrating quantum billiard corresponds to a $d$-term quantum system coupled in a time-dependent, self-consistent fashion to $r$ classical degrees-of-freedom. These classical dof correspond to the dov of the quantum billiard. Taking

$d=2$ and $r=1$, one obtains a system precisely analogous to a diatomic molecule (such as $\mathrm{NaCl}$ ) with two electronic states (of the same symmetry) coupled nonadiabatically by a single internuclear vibrational coordinate. 
Such symmetry, which can be expressed in terms of symmetry conditions in a superposition state's quantum numbers, 13, 6 are one possible cause of the electronic degeneracy discussed earlier. That is, degeneracy and neardegeneracy of electronic energy levels are often a consequence of a system's inherent symmetries. (It is important to note that there are also many systems in which the near-degeneracy of electronic eigenenergies is not caused by symmetry.)

Vibrations in more complicated molecules can have more nuclear degreesof-freedom, although because of constraints, they might not all be independent. Thus, only polyatomic molecules of certain forms are describable directly as vibrating quantum billiards. Others may be described in terms of other semiquantal models that depart somewhat from the vibrating billiard abstraction. These models nevertheless retain a semiquantal formulation analogous to that of vibrating quantum billiards. Additionally, there exist molecular systems for which the vibrating quantum billiard model is apt without adjustment. A two dov quantum billiard such as one with rectangular geometry with length $a$ and width $b$, for example, may be used to model nonadiabatic dynamics in a linear triatomic molecule with respective nuclear displacements $a(t)$ and $b(t)$ between the center atom and the two atoms to which it is bonded. Requiring left and right symmetry for all time-represented by the constraint $a(t) \equiv b(t)$-yields the quantum vibrating square, which has one dov.27 An $r$ dov quantum billiard may likewise be used to model nonadiabatic coupling between the nuclear and electronic states of a linear molecule consisting of a chain of $(r+1)$ atoms. Perhaps more physically relevant would be using an $r$ dov quantum billiard as a model for the cou- 
pling between electronic states and $r$ normal modes in a polyatomic molecule such as benzene. One can complicate the situation further by considering the coupling of polyatomic molecules by intermolecular forces such as hydrogen bonds. Such systems are describable as coupled vibrating quantum billiards and hence as coupled Schrödinger equations with time-dependent boundary conditions. To our knowledge, these systems have yet to be studied in this fashion.

For electronic-nuclear coupling, it is simplest to consider two-fold degeneracies, for which one uses two-mode Galërkin expansions. An example of such a degeneracy occurs in the Jahn-Teller $E \times e$ trigonal molecule, in which one doubly degenerate vibrational mode $(e)$ interacts with a (symmetryinduced) double electronic degeneracy $(E)$. This system has been analyzed by Whetten, Ezra, and Grant ${ }^{3}$ and by Zwanziger, Grant, and Ezra. back up a bit, however, and give a physical discussion of why Hamiltonian models of electronic-nuclear coupling are good qualitative approximations of reality. Note again that there are some systems for which even the abstract vibrating quantum billiard formulation (which is a toy model) is useful in this fashion.

The easiest situations to understand are ones in which the degenerate electronic energy levels are isolated energetically from other states. That way, one may neglect these other states, providing justification for the use of lowmode Galërkin expansions. Additionally, we assume that the molecule under consideration possesses strong restoring forces that prevent large-amplitude nuclear motions. Systems with these properties ordinarily arise from singlehole or single-electron degeneracies. One then examines the system quantum- 
mechanically in order to examine the coupling of electronic, vibrational, and rotational motion in these systems. One can examine the distribution of energy levels, for example, by considering correlation functions and limiting situations.

A hallmark of electronic degeneracy is the extreme sensitivity of rovibrational states to perturbations. The nuclear motions of a molecule and its coupling to electronic motion may be influenced heavily by the surrounding environment. Hence, in order to study individual rovibrational states (rather than the bulk properties of nonadiabatic systems of bound states), one must isolate the molecule of interest. This is a highly nontrivial proposition. Moreover, strong nonadiabaticity is rare in the lower rovibrational levels of ground-state electronic terms of most easily isolated molecules.3 For example, the ground states of stable molecules contain an even number of electrons with electronic components that are ordinarily fully symmetric. Open-shell polyatomic molecules with sufficient symmetry often also exhibit electronic degeneracies, but it is difficult to prepare such systems. Many transition metals have degenerate ground terms, although they are usually only observed in solutions or solids. At higher energies, such as those near the threshholds for chemical reactions or molecular dissociation, one expects to observe strong rovibrational motion. These situations, however, are not highly symmetric. They are also infested by complex, large-amplitude nuclear oscillations. Consequently, one cannot expect to examine the ground terms of stable molecules in order to experimentally observe few-state nonadiabatic motion.

Another situation worth considering involves excited molecular valence 
states, which display strong interactions between two or more electrons or holes. Many of the symmetric situations contain degenerate (or nearly degenerate) electronic energy levels. Spectroscopic examination of such systems often reveals degenerate molecular orbitals, so the excited states contain four or more related terms (of which two are degenerate). One can thus describe this electronic-nuclear coupling with a superposition state of four or more terms. One can similarly consider the ground states of molecules such as cyclobutadiene and cyclo-octatetraene, in which the two electrons of highest excitation arise from the the same degenerate molecular orbital.

A related application is the analysis of Jahn-Teller systems. In the presentation below, we borrow material from Vibronic Interactions in Molecules and Crystals by I. B. Bersuker and V. Z. Polinger 5 The Jahn-Teller theorem, which may be treated mathematically using the language of algebra and functional analysis, states that if the adiabatic potential of a system (which is a formal solution to the electronic part of the Schrödinger equation) has several crossing sheets, then at least one of these sheets has no extremum at the crossing point. Hence, degenerate (and even near-degenerate) electronic energy levels cannot be analyzed using the Born-Oppenheimer (adiabatic) approximation. As this approximation breaks down, one obtains nonadiabatic coupling between nuclear and electronic terms. That is, near such degeneracies and near-degeneracies, it is appropriate to use the semiquantal regime in order to study the dependence of the electronic eigenenergies on the system's nuclear degrees-of-freedom. According to the semiquantal approximation, this dependence is approximated as a continuum.

It may be apt to use the Jahn-Teller effect as a synonym for nonadiabatic 
coupling between nuclear and electronic systems, but it is conventional to apply this term more specifically. Hence, although vibrating quantum billiards exhibit behavior like the Jahn-Teller effect, it is not directly labeled as such. Additionally, we note that in the case of electronic near-degeneracies, the term pseudo-Jahn-Teller effect is sometimes used. Situations in which Jahn-Teller deformations have been observed include vibrations in crystals, numerous types of spectroscopy (NMR, Raman, etc.), multipole moments, the stereochemistry and instability of molecules, mechanisms of chemical reactions, and catalysis. Crystals that exhibit the Jahn-Teller effect (such as ferroelectric crystals) contrast strikingly with those that do not. For example, their structural phase transitions and elastic properties are different.

Effects analogous to Jahn-Teller distortion have been observed in other physical systems, including the pion-nucleon interaction in quantum field theory, the $\alpha$-cluster description of light nuclei, and the resonant interaction of light with matter. The formal analogy between pion-nucleon interaction in the static model of the nucleon 10 and the Jahn-Teller problem is used to apply the methods and ideas of scattering theory to Jahn-Teller distortions. One can also apply this analogy in reverse to study semiquantum chaos and nonadiabatic dynamics in pion-nucleon interactions, a special form of Yukawa coupling in quantum chromodynamics (QCD) 르 To our knowledge, this has not yet been done. Indeed, any system that exhibits Jahn-Teller deformation is expected to exhibit semiquantum chaos. If the system has three or more degrees-of-freedom, it may also exhibit semiquantum diffusion. 1 . 2

Jahn-Teller systems exhibit equivalent minima of adiabatic surfaces which correspond to several distorted nuclear configurations of equivalent symme- 
try. For example, a molecule of type $M L_{6}$ with a double electronic degeneracy in the regular octahedral configuration becomes elongated along one of its four-fold axes of symmetry $C_{4}$ because of the Jahn-Teller effect. There are three equivalent distortions because there are three axes with $C_{4}$ symmetry. (The set $C_{m}$ denotes the group of rotational symmetries of the $m$-gon. 39.43 Its elements are rotations by angle $2 \pi / m$, which are denoted $c_{m}$.) The Jahn-Teller theorem does not apply to double electronic spin degeneracies (Kramers degeneracies) or linear molecules. However, linear molecules with degenerate electronic eigenenergies are unstable with respect to bending distortions, which produces nonadiabatic behavior known as the Renner effect. Additionally, one dov quantum billiards may be used to describe nonadiabatic dynamics in diatomic (and hence linear) molecules. Though related to the Jahn-Teller effect, this nonadiabatic behavior is not precisely the same. It is more accurately termed the inverse Jahn-Teller effect because of the inverted dependence on the interatomic distance $a$. This leads to degeneracy in the adiabatic sheets at $a=\infty$ rather than at $a=0$ as in the Jahn-Teller effect. The consequences of this degeneracy may be observed in diatomic molecules when their interatomic displacement is large. As we have been stressing, electronic near-degeneracies are as important as actual degeneracies. In these near-degeneracies, two potential surfaces almost intersect but do not actually cross each other because of some sort of weak interaction near at the point of closest approach. The observation that such near-degeneracies are prevalent in diatomic molecules has been captured by the one dov quantum billiard model. An example of this phenomenon is in the near-intersection between the lowest ionic and covalent states of alkali 
halides. In $\mathrm{NaCl}$, this near-degeneracy occurs at large interatomic distanceswhich is consistent with our predictions. When the sodium and chloride ions are far apart from each other, the resonance integral for electron transfer between them is small. Hence, the smallness of the cross-term $H_{12}$ in the Hamiltonian is caused by the large difference in the electron distribution in the two electronic states. Analogous situations occur in more complicated polyatomic molecules. 4

Jahn-Teller effects are classified according to their tensorial construction, which describes their (nuclear and electronic) symmetries and degeneracies. The $E$ term refers to an orbital doublet (electronic double degeneracy). The canonical $E \times e$ Jahn-Teller effect describes the interaction of doubly-degenerate electronic states of representation $E_{k}$ term with doublydegenerate nuclear vibrations of representation $E_{2 k}$. . The representation $E_{m}$ has basis functions with the transformation properties $\psi_{ \pm m} \sim \exp ( \pm m \varphi)$, where $\varphi$ is an arbitrary rotation about the symmetry axis.) The simplest polyatomic systems in which this occurs are triangular molecules $X_{3}$, tetrahedral molecules $M L_{4}$, and octahedral molecules $M L_{6}$. The presence of nuclear degeneracies of this type lead to multmode $E \times(e+\cdots e)$ systems, where the number of factors of $e$ correspond to the number of vibrational modes of type $E$.

Another orbital-doublet Jahn-Teller system is denoted $E \times\left(b_{1}+b_{2}\right)$, which occur when electronic states transform as $\psi_{ \pm m} \sim \exp ( \pm i m \varphi / 4)$ in polyatomic systems with $C_{m}$ or $S_{m}(m=4 k)$ axes of symmetry. (The set $S_{m}$ denotes the group of rotary reflections in the $m$-gon. Its elements are given by $s_{m} \equiv \sigma_{h} c_{m}=p_{0} c_{2} c_{m}$, where $p_{0}$ represents parity (spatial inversion) 
and $\sigma_{h}$ is mirror reflection in the horizontal plane. 39 Rotary reflections are examples of improper rotations.) In the present situation, the active JahnTeller modes are the singlet low-symmetry displacements (one-dimensional representations) of type $B_{1}$ and $B_{2}$. Each $B_{i}$ represents an independent, nondegenerate vibrational mode. The simplest examples of $E \times\left(b_{1}+b_{2}\right)$ distortions occur in square planar and pyramidal molecules $M L_{4}$. One special case of interest, denoted $E \times b$, occurs when the vibronic coupling to one of the $B$ vibrational modes is neglible. The motion along the coordinate decoupled from the electronic dof consists of simple harmonic vibrations near the completely symmetric nuclear configuration.

The $T \times\left(e+t_{2}\right)$ Jahn-Teller distortion refers to the coupling of a triple electronic degeneracy $T$ with a doubly-degenerate nuclear vibration of type $E$ and a triply-degenerate nuclear mode of type $T$. The simplest cubic molecule that can exhibit such a distortion is $M L_{6}$, whose symmetry group is $O_{h}$. (The octahedral group $O_{h}=O \times C_{i}$ describes the full symmetry of a cube. The group $O$, consisting of 24 rotations, is the set of rotational symmetries of cubes and regular octahedrons. The group $C_{i}$ consists of the parity operator $p_{0}$ and the identity $I$.) Some important special cases are the $T \times e, T \times t_{2}$, $T \times d$, and $P \times d$ Jahn-Teller distortions. The adiabatic potential in the $T_{2}$ subspace has $O \cong T_{d}$ symmetry, where $T_{d}$ represents the full symmetry group of a tetrahedron. Type $D$ nuclear distortions describe the case of equal coupling of the electronic dof to the $E$ and $T_{2}$ nuclear modes at equal forcing $\omega \equiv \omega_{E}=\omega_{T}$ when the molecular Hamiltonian (describing the coupling between electronic and vibrational degrees-of-freedom) posesses $S 0(3)$ symmetry. (The parameter $\omega^{2}$ denotes the force constant of the normal vi- 
bration.) The type $P$ triple electronic degeneracy exhibits more symmetries than the type $T$ degeneracy. Therefore, the $P \times d$ distortion is a highly symmetric special case of the $T \times\left(e+t_{2}\right)$ Jahn-Teller systems. It occurs in cubic polyatomic systems in which the cubic splitting of $D$ mode of nuclear vibrations is negligible. ${ }^{\text {O }}$ That is, the $P$ configuration refers to three-fold degenerate $p$-orbitals $(j=0, m \in\{0, \pm 1\})$, and the $D$ configuration represents five-fold degenerate $d$-orbitals $(j=2, m \in\{0, \pm 1, \pm 2\})$. (Recall that $l$ and $m$ denote the orbital and azimuthal quantum numbers, respectively. 38 ) The $P \times d$ Jahn-Teller distortion then represents a triply degenerate nuclear vibration coupled to a five-dimensional electronic manifold in spherical symmetry. In going from spherical symmetry to octahedral symmetry, the $d$ orbitals split into $\left(e+t_{2}\right)$. The same coupling to $e$ and $t$ modes would then indicate a remnant of higher (spherical) symmetry. 39

Other Jahn-Teller effects include the $\Gamma_{8} \times\left(e+t_{2}\right)$ distortion and its special cases. Quadruplet terms ( such as $\Gamma_{8}$ ) occur in icosohedral systems as well as in cubic systems with spin-orbital coupling. (The term $\Gamma_{8}$ is the double-valued irreducible representation of the double group $\bar{O}^{2}$ of the rotation group of regular octahedrons $O$. Double groups are obtained by treating rotations by the angles $\alpha$ and $(\alpha+2 \pi)$ as different quantities even though they describe physically identical rotations, 39) The simplest situations with electronic quadruplets (corresponding to four-mode Galërkin expansions) occur in cubic polyatomic molecules which have an odd number of electrons. Icosohedral systems can also exhibit vibronic coupling of nuclear quadruplets and quintuplets (of types $u$ and $v$, respectively) with electronic quintuplets and quintuplets (of types $U$ and $V$, respectively). One possible interaction of 
these terms is $U \times(u+v)$, in which a quadruply degenerate electronic state is coupled both to quadruply and quintuply degenerate nuclear modes.

One can consider arbitrarily complicated Jahn-Teller distortions, even the simplest of which are dynamically interesting. Multiple distortions can occur in the same molecule or crystal. The individual distortions need not possess the same symmetry. Systems that exhibit multiple sets of Jahn-Teller distortions of a given symmetry are called multimode Jahn-Teller systems. Multiple distortions can occur, for example, in crystals with point defects, as their energy spectra contain discrete near-degenerate electronic eigenenergies well-separated from other energy levels. One can also observe multiple Jahn-Teller centers interacting with each other. Systems that exhibit such behavior are known as polynuclear clusters. For example, consider two octahedral complexes of type $M L_{6}$ can form a double-center Jahn-Teller system (a bioctahedron) in three different ways with the two central atoms lying on the common axes of symmetry of the second, third, or fourth order.

Both theoretical and experimental analyses have been vital to the study of nuclear-electronic coupling in molecules. Treatments with few active modesthat is, low-mode Galërkin projections-have been particularly useful, because they ease the analytical difficulty of the theory. One may then consider a fully quantum variational treatment of the nuclear part of the Hamiltonian, so that model parameters can be fit to experimental data. (One molecule for which this has been done is sym-triazine. 3) As one increases the order of the Galërkin approximation, however, the procedure becomes increasingly difficult both analytically and computationally. Hence, treating these situations in the fully quantum regime becomes untenable rather quickly. It is 
consequently useful to develop semiclassical and semiquantal techniques even for few-term superposition states in order to analyze molecular systems.

The present work describes a semiquantal technique, but a semiclassical theory (using the $\hbar \longrightarrow 0$ asymptotic formalism) of several-mode nuclear motion is worth studying as well. (Recall that the semiquantal regime is a semiclassical regime but not the "semiclassical" regime that is traditionally studied as part of the quantum mechanics curriculum.) If one considers this situation abstractly in terms of vibrating quantum billiards, then a semiclassical procedure does exist in principle. One quantizes the motion of the billiard boundary to obtain an example of quantum chaology (for which one uses the semiclassical limiting procedure). To our knowledge, however, this process has not actually been carried out for billiards with oscillating boundaries or for the physical situations described in the present paper.

\section{Solvated Electrons}

Vibrating quantum billiards may also be useful for the study of electron solvation, which describes the nonadiabatic process of the relaxation of excess electrons in fluids. In this discussion, we adapt the work of Space and Coker to this perspective. 6 . The equilibrium structure of electron solvation in numerous fluids involves the localization of excess electrons into a roughly spherical cavity within the solvent. This cavity is the boundary of our billiard, so describing the present situation with the radially vibrating spherical quantum billiard may be especially apt. The electron is treated quantummechanically, whereas the motion of the solvent is approximated as classical. Such systems are hence in the confines of the semiquantal regime. Short- 
range, repulsive electron-solvent interactions prevent solvent molecules from penetrating the billiard boundary into the region occupied by the electron.

There are a wide variety of relevant fluid types. Among the possibilities are simple hard-sphere liquids such as helium and exceptionally polar solvents like water and ammonia. Calculations have explored the dynamical rearrangements that solvent atoms must make in order to accomodate an injected electron as well as how the electron and solvent couple to provide various relaxation pathways. This description is consistent with recent analyses of vibrating quantum billiards, 13, 14, 25, 27

Equlibrium excess electronic states possess fairly well-defined symmetry, but solvated electronic states in an unperturbed fluid are more disordered and delocalized, as there is no well sufficiently deep to serve as an equilibrium trap. The electron has numerous near-degenerate local minima, so the system's eigenstates are combinations of functions centered in several different wells. States may thus have a positive density in more than one cavity, although the ground electronic state is usually in an $s$-like state of a single cavity. Recall that the canonical vibrating billiard model assumes boundary fluctuations that preserve some or all of the symmetries of the billiard. We thus surmise that such a model may be appropriate for equilibrium fluctuations, because in that situation one has a single cavity with many symmetries. The simplest model to use in this event would be the radially vibrating spherical quantum billiard. In order to account for the observed asymmetry prior to equilibrium, one may be able to extend the model by considering multiple-well potentials, higher-term Galërkin projections (which would account for larger degeneracies), multiple degrees-of-vibration, and-perhaps 
most importantly-coupled vibrating quantum billiards. All except the latter may be inserted into the model for a single vibrating billiard to obtain a more complicated single-cavity configuration. One would need to consider coupled billiards in order to capture the notion of multiple cavities.

When a solvated electron in the ground state of a solvent cavity is excited by light ("photoexcited") into various electronic states, one obtains nonadiabatic relaxation, which is suitably described using the semiquantal approximation. One may also explore the nonadiabatic relaxation that occurs when an excess electron is injected into various excited states of unperturbed fluid configurations. With such analysis, one may study the dynamical trapping processes that are responsible for the formation of the localized equilibrium state (in other words, the quantum billiard system).

As with polyatomic molecules, one uses the Born-Oppenheimer approximation to produce a semiquantal analysis. One obtains trajectories by solving classical equations of motion for the cavity. These evolution equations include forces due to the charge distribution of the currently occupied electronic state. Space and Cokere have shown that at least two types of nonadiabatic processes are important for solvated electron relaxation in simple fluids. The first of these processes is highly diabatic and may be illustrated by a pair of weakly coupled $p$-like orbital states in the same solvent cavity. Suppose that a $p_{y}$ state has a higher energy than a $p_{x}$ state because the cavity is not quite spherical. The cavity will then begin to elongate in the $y$-direction because of the influence of the charge distribution, which causes the energy gap between the two states to decrease. If the two states are weakly coupled, they mix only for small separations of their energy levels. 
Hence, appreciable mixing between these states occurs only in a small region of nuclear (solvent) configuration space. Consequently, the manifestation of this behavior is brief. In the present example, the diabatic transition occurs when the two adiabatic basis states (the two different types of $p$-orbitals) rotate into each other rapidly, which causes the occupied electron to hop between the two states. As a result, the occupied electron state, which is still causing the solvent cavity to expand in the $y$-direction, is now less energetic than the unoccupied $p_{x}$-orbital. There is never any drastic change in the electronic charge distribution.

Now consider highly diabatic relaxation in a more general context. is typically a nonzero electron density in more than one cavity in the fluid. This density exerts an outward forces on the surrounding solvent. Additionally, distinct states are coupled nonadiabatically (because they each have a density in the same trap). The nuclear velocities are initially uncoupled to the electronic forces, so the electron rapidly explores several closely-related regions before localizing in a single equilibrium density fluctuation. In a surface-hopping calculation, the excess electron hops between eigenstates that change fairly quickly with the solvent configuration (as the wells in the fluid are almost degenerate). The transitions in question are ordinarily not simple diabatic ones in which state identities change. Instead, they are strongly nonadiabatic, as the wavefunctions become a dynamically changing mixture of several states in closely-related solvent regions. This is a consequence of the lack of symmetry in the configuration space and phase space of the initial unperturbed solvent. Once the electron is localized to a single cavity, its transitions tend to be diabatic. It pushes out the surrounding sol- 
vent to an equilibrium state where the solvent-solvent and electron-solvent forces nearly balance. At this point, the canonical vibrating quantum billiard model is especially appropriate, as one now has the requisite symmetry preservation.

A second important nonadiabatic process occurs when two interacting electronic states are strongly coupled by the solvent dynamics. Suppose, for example, that the $p_{y}$ solvated-electron charge distribution considered above has continued its expansion of the solvent cavity along the $y$-axis. Simultaneously, solvent atoms near the cavity walls around the node in the $p_{y}$ wavefunction have begun to push into the cavity, which pinches off the middle region of the ground-state wavefunction, thereby causing it to increase in energy (since the relevant displacement parameter of the billiard is smaller when this happens). These two electron states occupy similar regions of space and are strongly coupled by the motion of the cavity boundary. The two associated energy levels may thus switch their ordering, as they can mix strongly even when there is a large energy disparity. When this occurs, the excess electronic potential due to the deforming cavity looks like a fluctuating double well. When the distorted $s$ and $p$ orbitals mix strongly, the solvated electron may be localized on either the left or right side of the double-well cavity. Eventually, either a left-well or right-well localization predominates, and the electron hops into a ground-state $s$-orbital.

The crux of the matter is that solvated electrons exhibit transient relaxation dynamics before they reach their equilibrium behavior. The canonical vibrating quantum billiard model may be useful for the equilibrium oscillations, whereas more complicated extensions of the model (especially coupled 
vibrating quantum billiards) may be appropriate to help describe several phases of the transient behavior. At short times, the occupied electron state is embedded in a continuum, so if the fluid density is sufficiently low, the electron can leak through the solvent and hop from state to state. At this point, the excess electronic absorption is characterized by a low-energy band. Once the solvated electron is in a particular cavity for a sufficiently long time interval, the diameter of the cavity increases rapidly, thereby localizing the solvated electron in a deeper well. The wall of the cavity still oscillates, but it is now reasonable to assume that its geometry is preserved under these vibrations, so that simple toy models such as vibrating quantum billiards may be useful.

\section{Buckb.paltsuangdaNanotubes}

It is well-known that the motion of electrons through quantum dots may be altered substantially by energy-level quantization and the charging of a single electron. Studies of electron transport have recently been extended to the realm of chemical nanostructures such as nanocrystals and nanotubes. A group of scientists at Lawrence Berkeley National Laboratory fabricated unimolecular transistors by connecting buckyballs to gold electrodes 10 Buckyballs, Time Magazine's molecule of the year in 1991, are $C_{60}$ molecules shaped like a soccer ball. The LBNL researchers then studied the nanomechanical vibrations of these buckyball transistors. They performed transport measurements that demonstrated coupling between the (nuclear) motion of the center-of-mass of the buckyball and the hopping of the single electron (electronic motion). This conduction mechanism was not observed previ- 
ously in quantum dot studies, although such electronic-nuclear coupling is a hallmark of nonadiabatic motion in molecular dynamics and the vibrating quantum billiard model.

The $C_{60}$ molecule is a fullerine that-like a soccer ball-has a surface consisting of twenty hexagons and twelve pentagons. Its geometry is thus more complicated than those that have been used in the study of vibrating quantum billiards, but it exhibits a similar type of nonadiabatic electronic-nuclear coupling. However, one can obtain a simpler model by approximating the buckyball as a sphere in order to study the coupling between its bouncing and the electronic motion. Perhaps one could then use perturbation theory to account for the more complicated geometry. Controlling such motion (and other types of motion!) in nanoscale objects is an important issue in the field of nanotechnology. Macroscopically, one may ignore the coupling of a rigid wall to a ball bouncing against it, but at sufficiently small scales one can no longer do this, a fact which is at the heart of the present review. In other words, when one studies objects at "new" scales (such as microscales and nanoscales), one must examine couplings between types of systems that have not traditionally been treated together as subcomponents of a single entity. Electronic-nuclear coupling heavily influences molecular motion, even though the electron mass is a small fraction of the molecular mass. The mechanical control of nanoscale objects ("NEMs") will allow smaller, faster, and more efficient versions of existing micro-electro-mechanic structures (MEMs) $111 \mathrm{~A}$ single-electron current can both detect and excite mechanical oscillations in a buckyball. For example, an electron with surplus energy precisely equal to the vibrational energy of the buckyball causes the buckyball to begin bounc- 
ing due to spontaneous emission of this energy. Furthermore, the electron continues to hop on and off the molecule. Electronic devices in which the electro-mechanical motion is so coupled could function as "electron turnstiles" that allow electrons to pass one at a time.

The various devices studied by Park, et al. exhibited a universal quantized excitation with an energy of about $5 \mathrm{meV}$. Such an excitation energy could arise in several possible manners in a single $C_{60}$ transistor. One hypothesis, which has been invoked in other nanosystems, is that this excitation energy is a result of highly excited electronic states of the buckyball. However, this possibility was dismissed for several reasons. The observed excitation energy was the same for both types of charge states of $C_{60}$, multiple excitations with the same spacing were observed, and this mechanism is not consistent with theoretical calculations of the electronic states of $C_{60}^{n-}$ ions.

A better explanation involves coupling between vibrational excitations of the buckyball with electronic tunnelling on and off $C_{60}$. That is, the authors proposed a nonadiabatic semiquantal system in which squishing couples to electronic hopping. In this nuclear mode, the buckyball deforms ("squishes") a little bit like a ball being pressed against the ground. With this mechanism, the observation of multiple $\partial I / \partial V$ features with identical spacing would then result from the excitation of integral numbers of vibrational quanta. These vibrational modes, moreoever, would be identical for both charge states of the buckyball. However, there are some problems with this explanation as well. The internal vibrational mode of lowest energy is about $35 \mathrm{meV}$, so we require a different explanation. The lowest pulsing internal mode, like what occurs in the radially vibrating spherical quantum billiard, is even more energetic. 
A possible resolution involves another type of electronic-nuclear coupling. One hypothesizes a mechanism in which oscillations of the buckyball's centerof-mass within a confinement potential bind it to a gold surface, thereby predicting a vibrational energy of about $5 \mathrm{eV}$ (as was observed experimentally). Hence, the following nanomechanical motion is predicted: an electron jumps onto a $C_{60}^{n-}$ molecule, which causes an attraction between the additional electron and its image charge on the gold. This electrostatic interaction pulls the buckyball ion closer to the gold surface and results in mechanical motion of $C_{60}$, like that of a soccerball bouncing against the ground. Although slightly different from the mechanisms we have been discussing (which correspond more closely to the second hypothesis), this third hypothesis also predicts nonadiabatic electronic-nuclear coupling, for which a semiquantal description is appropriate. It is amenable to the same analyses that have been performed on vibrating quantum billiards, but the relevant Hamiltonian takes a slightly different form.

The dynamical situation in the bouncing buckyball is reminiscent of the Franck-Condon process that occurs in electron transfer and light absorption in molecules. In these situations, the electronic motion is accompanied by vibrational excitation. The transport measurements discussed above may be used both to probe and to excite molecular motion. In particular, the buckyball transistor that was studied behaved as a high-frequency nanomechanical oscillator. The electronic component of its oscillations may be quantized so that the system is treatable in the semiquantal regime. This sort of coupling between quantized electronic (fast) and unquantized mechanical (slow) degrees-of-freedom should become important for electron transport through 
nanomechanical systems such as buckyballs and carbon nanotubes. The difference between this system and a vibrating quantum billiard of appropriate geometry is that the nuclear motion is described by a "bouncing" of the center-of-mass rather than a pulsing of the billiard boundary. One could obtain a reasonable toy model with an appropriate nuclear Hamiltonian.

More generally, one can consider the bouncing mode on top of the buckyballs internal modes. If one considers only bouncing, one obtains a one dof Hamiltonian. This nuclear degree-of-freedom, which describes the height of the ball, is coupled to a single electronic state. In this exactly-solvable model, one treats the nucleus as a point mass. The nuclear Hamiltonian consists of the harmonic potential plus a second term which contributes nothing when the electron is off the buckyball and shifts the oscillator when it is on it. That is, the nuclear Hamiltonian $H_{N}$ takes the form

$$
H_{N}=\frac{1}{2} k\left(z-z_{0}\right)^{2}+e \mathcal{E} z \hat{n},
$$

where $k$ is a spring constant, $z$ is the displacement of the buckyball, $e$ is the charge of an electron, $\mathcal{E}$ is the electric field, and the operator $\hat{n}$ is 1 when the electron is on the buckyball and 0 when it is not. The electronic Hamiltonian is

$$
H_{e}=E_{0}-e \mathcal{E} z
$$

where $E_{0}$ is the energy of the electron without the coupling. One can then complicate matters by modeling the geometry of the buckyball as spherical. Internal nuclear modes then give additional nuclear dof, and their coupling to multiple electronic states is also relevant. That is, when considering the 
internal modes of the buckyball, it becomes important to consider multiple electronic states of the molecule rather than just one. When treating the buckyball as a point mass, it was sufficient to consider only the ground state in the analysis of its because the excited states are not energetically close. Nevertheless, a study considering multiple-term superposition states of a zero-dimensional bouncing ball might still be illuminating. When the buckyball is three-dimensional rather than zero-dimensional, its (nuclear) deformation can lead smaller separations in the eigenenergies, thereby forcing one to consider Galërkin projections of more than one electronic state. However, the number of states that should be considered is open to debate. To answer this question, one must consider the buckyball's electronic spectrum. Moreover, the appropriate number of electronic states will depend on the internal (Jahn-Teller) distortions under consideration. One can complicate matters further by considering the buckyball's true soccerball geometry rather than a spherical approximation. Unlike a stationary spherical quantum billiard, a billiard shaped like a soccerball is no longer globally separable, which leads to a marked increase in the complexity of the system's internal

dynamics.14 It is important to note that these internal Jahn-Teller modes can be studied without the bouncing dynamics-which essentially become an extra degree-of-freedom. A buckyball is expected to exhibit nonadiabatic behavior even without the bouncing mode.

\subsection{Nanotubes}

Carbon nanotubes have been studied extensively during the past decade from both scientific and engineering perspectives, as they offer numerous potential technological applications. 
cylinders with very large aspect ratios. That is, their length is much larger than their cross-sectional radius, so there are some respects in which they can be viewed as one-dimensional objects. However, nanotubes also have hemispherical caps, so they are not truly cylindrical objects (although they possess the same reflection and rotation symmetries). For now, we ignore this and treat them as cylinders. We will briefly revisit this issue later and discuss the utility of studying carbon nanotubes using the more accurate "pillbox" geometry. Carbon nanotubes may be studied as quantum billiards because, like quantum dots, they exhibit ballistic electron transport 4 国 In particular, there is evidence that electrons may traverse the length of some single-walled carbon nanotubes ballistically without significant scattering. Such devices may thus be thought of as resonant cavities for electrons in which the nanotube acts as a waveguide. Additionally, the contacts between the nanotube and the electrodes act as weakly reflecting barriers.6

Under the assumption of a cylindrical geometry, every nanotube is specified by its diameter and the chirality of the rows of carbon atoms relative to the axis of the cylinder. Their lengths range from the micrometer to milimeter scales, and the length of a given nanotube may undergo oscillations. Nanotube diameters, which may also vibrate, range from about 0.7 to $1.6 \mathrm{~nm}$. (In contrast, buckyballs have a diameter of about $0.72 \mathrm{~nm}$.) Because of these radial and longitudinal vibrations and the relevance of the semiquantal regime to the present situation, carbon nanotubes are potentially describable as two dov vibrating quantum billiards. 14.27 That is, they have two nuclear degrees-of-freedom that couple to electronic motion. This description of nanotubes has not yet been studied, and it may prove bene- 
ficial to do so. Another vibrational mode resembles distortions reminiscent of plucking a guitar string in which the entire nanotube oscillates back and forth while retaining its shape.

Another possible model of carbon nanotubes is a quantum billiard shaped like a pillbox, whose geometry consists of a cylinder adjoined to two spherical caps. Thus, unlike the cylindrical quantum billiard, this system is not globally separable.14 Consequently, even a stationary pillbox quantum billiard, whose longitudinal cross section is a stadium quantum billiard, 28 experiences quantum signatures of classical chaos ("quantum chaology"), much like the quantum Sinai billiard 1, 25, 28, 49 Therefore, there is a tangible difference between this model and the cylindrical quantum billiard, as the latter is integrable unless the boundary oscillates. Analogous to the situation with buckyballs, one can treat the pillbox geometry as a perturbation of the cylindrical one.

One aspect of nanotube dynamics that has been studied is nonlinear resonance effects and their relation to positional instability. 6 Such resonances provide evidence that the upper and lower limits of nanotube diameters may be affected significantly by the system's internal dynamics. Sufficiently chaotic motion during the attempted formation of a nanotube could preclude organization into such a well-formed structure. As with other polyatomic molecules, one may study the nonlinear dynamics of carbon nanotubes using the classical trajectory method. It has been shown that onset of unstable motion occurs rapidly when certain vibrational modes are coupled. This large-amplitude motion is caused by low-order nonlinear resonances. The dynamics of the nanotube were observed to depend sensitively on the length of their diame- 
ters. It was also observed that the dependence of the positional stability on the diameter was correlated with the length of the nanotube, thereby implying a dependence of the onset of large-amplitude motion on the aspect ratio of the device. As in many other fields of science, 5 these dependencies obey scaling laws.

Positional instability may arise from strong coupling between longitudinal and radial ("ring-breathing") modes. Sumpter and Noid 8 found that the two modes had a 1:2 frequency ratio, which is sometimes called Fermi resonance and is one of the stronger forms of nonlinear coupling. Such resonances have been attributed to the onset of readily manifested energy transfer in several polyatomic and macromolecules. In the present situation, rapid energy transfer between low-frequency modes (which retain their energy because of resonant transfer between longitudinal and radial motion) causes small-diameter carbon nanotubes to become unstable in the sense that they exhibit large-amplitude motion. Additionally, nanotubes are expected to exhibit Jahn-Teller distortions that could significantly affect their electronic structure, 9 so a nonadiabatic semiquantal description should be appropriate to study the dynamics of these devices. Quantum billiards may also be useful to study other devices such as the horn-shaped nanobugles (that are also carbon-based) and the silicon-based nanocages that can be formed surrounding a metal ion.

\section{Other Applications}

There are numerous other systems in which semiquantum chaos occurs. For example, consider a collection of atoms in a resonant cavity interacting 
self-consistently with the electromagnetic field within the cavity 12 Berman, Bulgakov, and Zaslavsky 5 studied this system quasiclassically to show that the quasiclassical approximation may break down in a shorter time frame for a system whose classical limit $(\hbar=0)$ is chaotic than it does for one whose limit is integrable. In the quasiclassical regime, the quantity

$$
\frac{\hbar}{I} \ll 1
$$

(The variable $I$ is a characteristic action of the problem.) For integrable systems, the approximation breaks down for time $\tau_{\hbar}$ given by

$$
\tau_{\hbar}=C / \hbar
$$

where $C$ is a constant. For chaotic systems, however, the breakdown time is instead about

$$
\tau_{\hbar}=C \ln \left(\frac{C}{\hbar}\right)
$$

Ideally, one studies the dynamics of atoms in a resonant cavity by analyzing two electronic states (i.e., using a two-mode Galërkin expansion). Suppose that the electromagnetic field has a single mode whose frequency equals the transition frequency of the atoms. (This situation is encompassed by a model attributed to Dicke.) This system was approximated quasiclassically using a resonance approximation, which provides a basis for understanding the dynamics of many problems involving the interaction of atoms and fields. (One can also treat this system semiquantally.) In their analysis, Berman, Bulgakov, and Zaslavsky constructed a theory to obtain the quantum corrections for the dynamics of this resonant cavity system. In particular, they derived equations of motion for the quantum-mechanical expectation values 
and quantum correlation functions. They demonstrated numerically that the nature of the growth of the quantum corrections depended very strongly on whether the classical limit was integrable or chaotic. This underscores a very important aspect of quantum chaos: one obtains systems that behave in a fundamentally different manner depending on whether one has quantized a classically chaotic or classically integrable system.

Another situation in which the semiquantal approximation is used to analyze nonadiabatic dynamics is in collective nuclear motion. 19 Near levelcrossings, in which the relative energy between two eigenstates changes sign, there are several effects that can occur during the time evolution of the slow (nuclear) variables. Among them are Landau-Zener transitions, the molecular Aharanov-Bohm effect, and geometric phase, a non-integrable quantum phase that we discussed previously. Moreover, the concepts of quantum chaos and level crossings go hand in hand, which the reader may have already gathered from prior discussions in this review. Traditional studies of large-amplitude collective motion do not discuss phenomena such as Berry phase, despite the fact that microscopic computable quantities such as potential energies vary rapidly (or may even be singular or nearly so) when sufficiently close to level crossings. Bulgad 19 analyzed a simple model to illustrate the effects of level crossings on bound nuclear collective motion.

Another relevant system is a quantum spin $\vec{\sigma}$ interacting with the motion of a particle.17 The Hamiltonian of one such system is

$$
H=B \sigma_{z}+C x \sigma_{x}+\frac{p^{2}}{2 m}+V(x)
$$

where the first term is the spin Hamiltonian, the last two terms are the particle Hamiltonian, and the second term represents the interaction between 
particle and spin. This system has been explored in both the fully quantum and semiquantum regimes.

Nonadiabatic coupling in semiquantal physics is also relevant to the study of micromasers 16 Consider a single two-level atom in a cavity that is coupled to a "high $Q$ " cavity mode. (High $Q$ regimes are ones in which the cavity has a small number of modes interacting with the atomic system.) This regime is relevant, for example, to superconducting microwave cavities and extremely high finesse optical resonators.) Suppose that an atom, initially in an excited state $e$, interacts with an initially empty cavity for a time $T$ before exiting. Moreover, suppose that another excited electron enters the cavity before the resultant field from the previous electron has had time to relax. This second electron is consequently affected both by the field and the cavity. Suppose that this process continues ad infinitum. In a steady-state operation, the field in the cavity results from competition between relaxation and the interaction with successive electrons. The system so obtained is a micromaser, which is easily seen to be amenable to a semiquantal description.

Finally, consider radio frequency superconducting quantum-interferencedevice (SQUID) magnetometers 18 Such systems consist of a superconducting weak link ring and an $L C$ oscillator circuit (a tank circuit), which is driven by an external source of current (ordinarily at radio frequencies). The current leads to a magnetic flux in the inductor, which interacts with the SQUID ring via a mutual inductance $M$. This coupled magnetic flux induces a screening current in the ring that is also coupled to the tank circuit. This model exhibits chaotic dynamics, which has also been reported in experimental rf-SQUID systems. To incorporate quantum-mechanical information into 
this model, one quantizes the motion of the ring. One thereby obtains a semiquantal system with a quantum component (the ring) coupled to a linear classical oscillator (the tank circuit). This system has been shown to exhibit semiquantum chaos.

\section{Conclusions}

Every physical regime is an approximation of reality in some form or another. One lesser-known regime is the semiquantal one, which may be used to describe systems with both classical and quantum subcomponents. In the present review, we discussed nonadiabatic dynamics in the semiquantal regime. We focussed on the arena of electronic-nuclear coupling in molecular dynamics, but we also included examples from several other situations. We formulated the notion of semiquantal physics and then discussed nonadiabatic phenomena, concentrating on their relation to the Born-Oppenheimer approximation. We also discussed several systems in which such behavior can occur.

\section{Acknowledgements}

I would like to thank Greg Ezra for carefully reading an early version of this manuscript, helping me correct several mistakes, and improving my understanding of physical chemistry with explanations and references to numerous journal articles. I would also like to than Paul McEuen for useful discussions during the preparation of this manuscript. Additionally, Greg Colyer alerted me to the study of chaotic dynamics in SQUIDs. Finally, the

referees made several excellent suggestions that improved the exposition in 
this paper.

\section{References}

[1] M. C. Gutzwiller, Chaos in Classical and Quantum Mechanics, Number 1 in Interdisciplinary Applied Mathematics, Springer-Verlag, New York, NY, 1990.

[2] J. J. Sakurai, Modern Quantum Mechanics, Addison-Wesley Publishing Company, Reading, MA, Revised edition, 1994.

[3] R. L. Whetten, G. S. Ezra, and E. R. Grant, Annual Reviews of Physical Chemistry 36, 277 (1986).

[4] J. W. Zwanziger, E. R. Grant, and G. S. Ezra, Journal of Chemical Physics 85, 2089 (1986).

[5] I. B. Bersuker and V. Z. Polinger, Vibronic Interactions in Molecules and Crystals, Number 49 in Springer Series in Chemical Physics, SpringerVerlag, New York, NY, 1989.

[6] B. Space and D. F. Coker, Journal of Chemical Physics 94, 1976 (1991).

[7] B. Space and D. F. Coker, Journal of Chemical Physics 96, 652 (1992).

[8] B. G. Sumpter and D. W. Noid, Journal of Chemical Physics 102, 6619 (1995).

[9] M. Menon, E. Richter, and K. R. Subbaswamy, Journal of Chemical Physics 104, 5875 (1996). 
[10] H. Park et al., Nature 407, 57 (2000).

[11] L. Kouwenhoven, Nature 407, 35 (2000).

[12] R. Blümel and B. Esser, Physical Review Letters 72, 3658 (1994).

[13] R. L. Liboff and M. A. Porter, Chaos 10, 366 (2000).

[14] M. A. Porter and R. L. Liboff, International Journal of Bifurcation and Chaos (To appear September, 2001).

[15] G. P. Berman, E. N. Bulgakov, and G. M. Zaslavsky, Chaos 2, 257 (1992).

[16] S. Haroche, Cavity quantum electrodynamics, in Fundamental Systems in Quantum Optics, edited by J. Dalibard, J.-M. Raimond, and J. ZinnJustin, pages 767-940, Elsevier Science Publishers B. V., 1992.

[17] L. E. Ballentine, Physical Review E 63, 1 (2001).

[18] J. Diggins et al., Physical Review E 49, 1854 (1994).

[19] A. Bulgac, Physical Review Letters 67, 965 (1991).

[20] M. E. Peskin and D. V. Schroeder, An Introduction to Quantum Field Theory, Perseus Books, Cambridge, Massachusetts, 1995.

[21] P. Pechukas, Physical Review 181, 166 (1969).

[22] P. Pechukas, Physical Review 181, 174 (1969).

[23] D. F. Coker and L. Xiao, Journal of Chemical Physics 102, 496 (1995). 
[24] G. Baym, Lectures on Quantum Mechanics, Lecture Notes and Supplements in Physics, Perseus Books, Reading, Massachusetts, 1990.

[25] M. A. Porter and R. L. Liboff, Discrete and Continuous Dynamical Systems , 310 (2001), Proceedings of the International Conference on Dynamical Systems and Differential Equations: Georgia, May 18-21, 2000.

[26] H.-D. Meyer and W. H. Miller, Journal of Chemical Physics 70, 3214 (1979).

[27] M. A. Porter and R. L. Liboff, International Journal of Bifurcation and Chaos (To appear September, 2001).

[28] R. Blümel and W. P. Reinhardt, Chaos in Atomic Physics, Cambridge University Press, Cambridge, England, 1997.

[29] L. Allen and J. H. Eberly, Optical Resonance and Two-Level Atoms, Dover Publications, Inc., New York, NY, 1987.

[30] A. Friedman, Notices of the American Mathematical Society 47, 854 (2000).

[31] M. A. Porter and R. L. Liboff, International Journal of Bifurcation and Chaos 11, 903 (2001).

[32] R. P. Feynman, Physical Review 56, 340 (1939).

[33] R. Temam, Infinite-Dimensional Dynamical Systems in Mechanics and Physics, Number 68 in Applied Mathematical Sciences, Springer-Verlag, New York, NY, 2nd edition, 1997. 
[34] J. E. Marsden and T. S. Ratiu, Introduction to Mechanics and Symmetry, Number 17 in Texts in Applied Mathematics, Springer-Verlag, New York, NY, second edition, 1999.

[35] J. W. Zwanziger, M. Koenig, and A. Pines, Annual Reviews of Physical Chemistry 41, 601 (1990).

[36] R. Abraham, J. E. Marsden, and T. Ratiu, Manifolds, Tensor Analysis, and Applications, Number 75 in Applied Mathematical Sciences, Springer-Verlag, New York, NY, 2nd edition, 1988.

[37] M. P. do Carmo, Riemannian Geometry, Birkhäuser, Boston, MA, 1992.

[38] R. L. Liboff, Introductory Quantum Mechanics, Addison-Wesley, San Francisco, CA, 3rd edition, 1998.

[39] T. Inui, Y. Tanabe, and Y. Onodera, Group Theory and Its Applications in Physics, Number 78 in Solid-State Sciences, Springer-Verlag, Berlin, Germany, 1990.

[40] J. F. Cornwell, Group Theory in Physics, volume 2, Harcourt Brace Jovanovich, Publishers, London, England, 1984.

[41] A. J. Lichtenberg and M. A. Lieberman, Regular and Chaotic Dynamics, Number 38 in Applied Mathematical Sciences, Springer-Verlag, New York, NY, 2nd edition, 1992.

[42] G. Haller, Chaos Near Resonance, Number 138 in Applied Mathematical Sciences, Springer-Verlag, New York, NY, 1999. 
[43] D. S. Dummit and R. M. Foote, Abstract Algebra, Prentice Hall, Englewood Cliffs, NJ, 1991.

[44] G. Herzberg and H. C. Longuet-Higgins, Discussions of the Faraday Society 35, 77 (1963).

[45] L. Kouwenhoven and C. Marcus, Physics World, 35 (1998).

[46] C. T. White and T. N. Todorov, Nature 411, 649 (2001).

[47] W. Liang et al., Nature 411, 665 (2001).

[48] S. W. MacDonald and A. N. Kaufman, Physical Review A 37, 3067 (1988).

[49] A. Katok and B. Hasselblatt, Introduction to the Modern Theory of Dynamical Systems, Cambridge University Press, New York, NY, 1995.

[50] M. Schroeder, Fractals, Chaos, Power Laws: Minutes from an Infinite Paradise, W. H. Freeman and Company, New York, NY, 1991. 